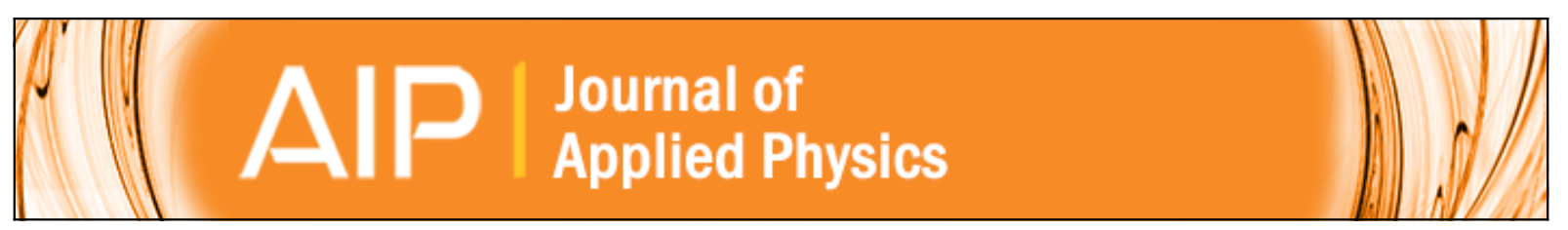

\title{
Electronic structure and thermoelectric properties of pnictogen-substituted ASn1.5Te1.5 (A=Co, Rh, Ir) skutterudites
}

Alex Zevalkink, Kurt Star, Umut Aydemir, G. Jeffrey Snyder, Jean-Pierre Fleurial, Sabah Bux, Trinh Vo, and Paul von Allmen

Citation: Journal of Applied Physics 118, 035107 (2015); doi: 10.1063/1.4926479

View online: http://dx.doi.org/10.1063/1.4926479

View Table of Contents: http://scitation.aip.org/content/aip/journal/jap/118/3?ver=pdfcov

Published by the AIP Publishing

\section{Articles you may be interested in}

Thermoelectric properties of DC-sputtered filled skutterudite thin film

J. Appl. Phys. 117, 125304 (2015); 10.1063/1.4916238

Influence of substituting $\mathrm{Sn}$ for $\mathrm{Sb}$ on the thermoelectric transport properties of CoSb3-based skutterudites J. Appl. Phys. 115, 103704 (2014); 10.1063/1.4867609

Thermoelectric performance of p-type skutterudites $\mathrm{Yb} x$ Fe 4-yPt y Sb12 (0.8 $\leq \mathrm{x} \leq 1, \mathrm{y}=1$ and 0.5$)$

J. Appl. Phys. 113, 143708 (2013); 10.1063/1.4800827

Thermoelectric properties of indium filled and germanium doped Co4Sb12 skutterudites

J. Appl. Phys. 111, 023708 (2012); 10.1063/1.3677982

Substitution effect on the thermoelectric properties of $\mathrm{p}$-type half-Heusler compounds: $\mathrm{Er} \mathrm{Ni} 1-\mathrm{x} \mathrm{Pd} \times \mathrm{Sb}$ J. Appl. Phys. 104, 013714 (2008); 10.1063/1.2956699

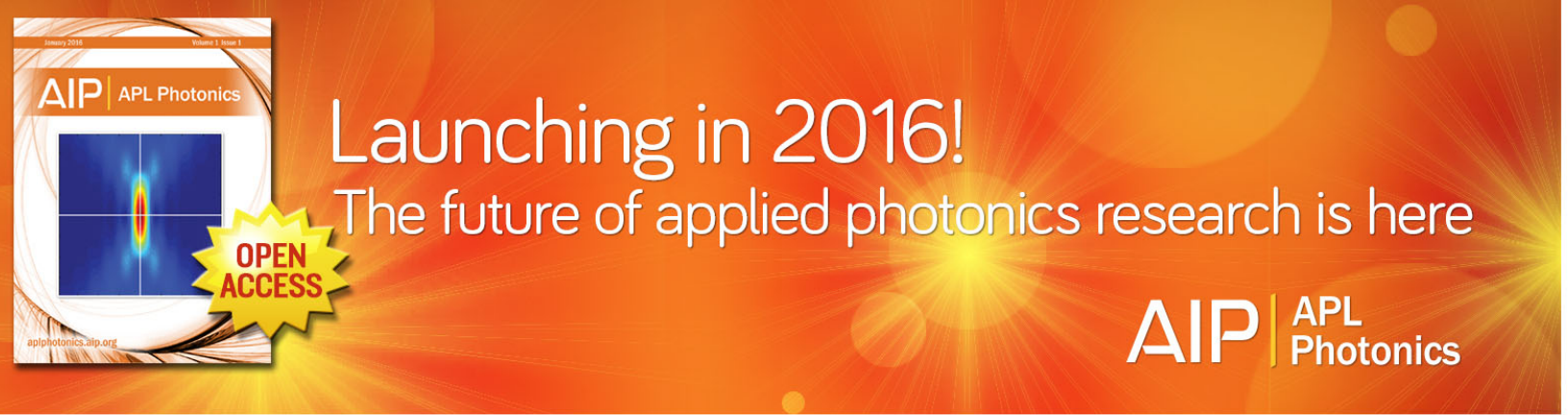




\title{
Electronic structure and thermoelectric properties of pnictogen-substituted $A \mathrm{Sn}_{1.5} \mathrm{Te}_{1.5}(A=\mathrm{Co}, \mathrm{Rh}, \mathrm{Ir})$ skutterudites
}

\author{
Alex Zevalkink, ${ }^{1,2, a)}$ Kurt Star, ${ }^{1, a)}$ Umut Aydemir, ${ }^{2}$ G. Jeffrey Snyder, ${ }^{2}$ Jean-Pierre Fleurial, ${ }^{1}$ \\ Sabah Bux, ${ }^{1}$ Trinh Vo, ${ }^{3}$ and Paul von Allmen ${ }^{3}$ \\ ${ }^{1}$ Thermal Energy Conversion Technologies Group, Jet Propulsion Laboratory, 4800 Oak Grove Drive, \\ Pasadena, California 91109, USA \\ ${ }^{2}$ Department of Materials Science, California Institute of Technology, 1200 E California Blvd, Pasadena, \\ California 91125, USA \\ ${ }^{3}$ Instrument Software and Science data systems Group, Jet Propulsion Laboratory, 4800 Oak Grove Drive, \\ Pasadena, California 91109, USA
}

(Received 7 April 2015; accepted 27 June 2015; published online 21 July 2015)

\begin{abstract}
Substituting group 14 and 16 elements on the pnictogen site in the skutterudite structure yields a class of valence-precise ternary $A X_{1.5} Y_{1.5}$ compounds $(A=\mathrm{Co}, \mathrm{Rh}, \mathrm{Ir}, X=\mathrm{Sn}, \mathrm{Ge}$, and $Y=\mathrm{S}, \mathrm{Se}$, $\mathrm{Te}$ ), in which $X$ and $Y$ form an ordered sub-structure. Compared with unfilled binary skutterudites, pnictogen-substituted phases exhibit extremely low lattice thermal conductivity due to increased structural complexity. Here, we investigate the role of the transition metal species in determining the electronic structure and transport properties of $A \mathrm{Sn}_{1.5} \mathrm{Te}_{1.5}$ compounds with $A=\mathrm{Co}$, Rh, Ir. Density functional calculations using fully ordered structures reveal semiconducting behavior in all three compounds, with the band gap varying from 0.2 to $0.45 \mathrm{eV}$. In $\mathrm{CoSn}_{1.5} \mathrm{Te}_{1.5}$, the electronic density of states near the gap is significantly higher than for $A=\operatorname{Ir}$ or Rh, leading to higher effective masses and higher Seebeck coefficients. Experimentally, Ir and Rh samples exhibit relatively large $p$-type carrier concentrations and degenerate semiconducting behavior. In contrast, $\mathrm{CoSn}_{1.5} \mathrm{Te}_{1.5}$ shows mixed conduction, with $n$-type carriers dominating the Seebeck coefficient and light, high mobility holes dominating the Hall coefficient. $z T$ values of up to 0.35 were obtained, and further improvement is expected upon optimization of the carrier concentration or with $n$-type doping. (C) 2015 AIP Publishing LLC. [http://dx.doi.org/10.1063/1.4926479]
\end{abstract}

\section{INTRODUCTION}

Thermoelectric generators are currently useful in a variety of specialized applications to convert waste heat energy into electric power. ${ }^{1}$ Their widespread use, however, remains limited by the efficiency of thermoelectric materials, which is governed by the figure of merit, $z T=\frac{\sigma \alpha^{2} T}{\kappa}$. Here, $\alpha$ is the Seebeck coefficient, $\sigma$ is the electronic conductivity, and $\kappa$ is thermal conductivity. Optimization of $\alpha$ and $\sigma$ can be achieved by tuning the carrier concentration, while the lattice contribution to $\kappa$ can be targeted by scattering or slowing phonons. $^{2-4}$ Binary skutterudite compounds with the general formula $\mathrm{APn}_{3}(A=\mathrm{Co}, \mathrm{Rh} \text {, or } \mathrm{Ir} \text {, and } \mathrm{Pn}=\mathrm{P}, \mathrm{As} \text {, or } \mathrm{Sb})^{5,6}$ crystallize in a body-centered cubic structure $(\operatorname{Im} \overline{3})$ characterized by large voids. Although they exhibit extremely high electronic mobility, their thermoelectric performance suffers due to high lattice thermal conductivity. ${ }^{5,6}$ To date, the most successful strategy for reducing $\kappa_{L}$ in skutterudites has been to partially fill the voids in the structure with a wide variety of elements, including alkali and alkaline earth metals, rare earth metals, and transition metals. ${ }^{7,8}$ These loosely bonded filler atoms form low frequency vibrational modes that can both scatter and reduce the velocity of acoustic phonons, ${ }^{9-13}$ leading to large reductions in $\kappa_{L}$. In many cases, the addition of filler atoms has enabled high $z T$ values. ${ }^{14,15}$

\footnotetext{
${ }^{\text {a) }}$ A. Zevalkink and K. Star contributed equally to this work.
}

Recently, an alternative strategy for reducing $\kappa_{L}$ has emerged in the form of ternary $A X_{1.5} Y_{1.5}$ skutterudite compounds ( $A=\mathrm{Co}$, Rh, Ir, $X=\mathrm{Sn}, \mathrm{Ge}, Y=\mathrm{S}$, Se, Te), in which the group 15 pnictogen site is substituted by equal parts group 14 and group 16 elements. Crystallographic studies have shown that $X$ and $Y$, rather than being randomly distributed on the pnictogen site, are ordered in layers perpendicular to the [111] direction, residing at opposite corners of the 4-cornered rings, as shown in Figure 1. ${ }^{16-21}$ Ordering leads to distortion of the anion sub-lattice, thus reducing the symmetry of the structure from cubic to rhombohedral (space group $R \overline{3}$ ). Order-disorder transitions have been observed in $\mathrm{CoGe}_{1.5} Y_{1.5}(Y=\mathrm{S}, \mathrm{Te})$ at high temperatures, but the potential impact that such a transition has on the transport properties is not yet well understood.

The thermoelectric properties of ordered $A X_{1.5} Y_{1.5}$ compounds have been investigated in several experimental and computational studies. ${ }^{6,17-19,22-25}$ As expected from their valence-precise electron count, they are generally found to exhibit non-degenerate semiconducting behavior. Compared with binary skutterudites, high Seebeck coefficients and low electronic conductivities were observed, suggesting larger effective masses. This was supported by a recent computational study of $\operatorname{Co} X_{1.5} Y_{1.5}$ compounds, which revealed heavier bands and larger band gaps in pnictogen substituted skutterudites compared with the binary analogues. ${ }^{26}$ As anticipated, the added unit cell complexity leads to lower lattice thermal conductivities than in binary skutterudites, while 


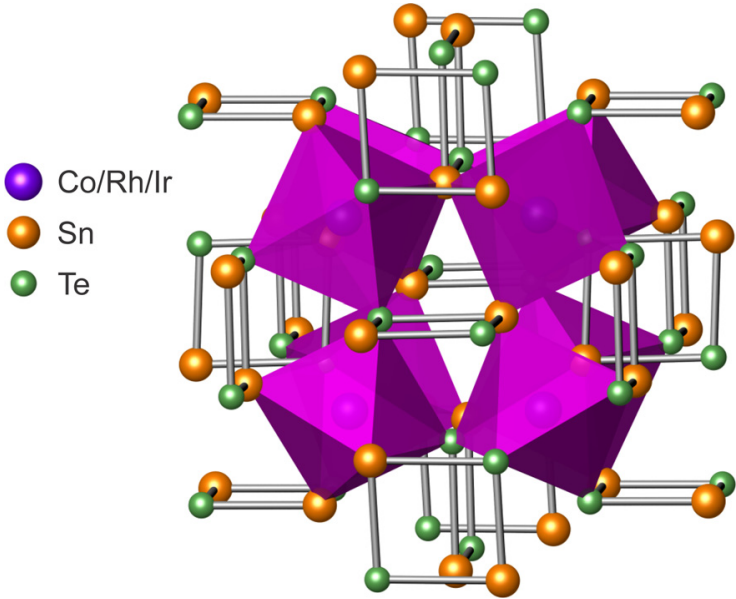

FIG. 1. $A \mathrm{Sn}_{1.5} \mathrm{Te}_{1.5}$ pnictogen substituted skutterudites contain octahedrally coordinated transition metal species $(A=\mathrm{Co}, \mathrm{Rh}, \mathrm{Ir})$ and $\mathrm{Sn}$ and Te residing in opposite corners of square rings. These are distorted relative to the $\mathrm{Sb}_{4}$ rings found in binary $\mathrm{ASb}_{3}$ skutterudites, leading to reduced symmetry (space group $R \overline{3}$ ).

further $\kappa_{L}$ reductions can be achieved with fillers. ${ }^{17,23,25}$ However, high $z T$ values have not yet been achieved (maximum of $z T=0.16$ in Ni-doped $\operatorname{CoSn}_{1.5} \mathrm{Se}_{1.5}$ ), due in part to lack of optimization of the electronic properties, which have been overly insulating in most cases. ${ }^{18}$

With the exception of studies reported by Bos et al. ${ }^{20}$ and Fleurial et al., ${ }^{6}$ very little attention has been given to the densest examples of pnictogen substituted skutterudites (i.e., $A \mathrm{Sn}_{1.5} \mathrm{Te}_{1.5}$ or Ir- and Rh-based compounds) partly because of the high cost of these elements. For space applications, however, performance requirements such as efficiency and thermal stability outweigh cost and the potential for very low thermal conductivity in such compounds provides sufficient motivation to investigate their thermoelectric properties. Here, we use a combination of density functional calculations and high temperature transport measurements to characterize the thermoelectric properties of $A \mathrm{Sn}_{1.5} \mathrm{Te}_{1.5}$ skutterudite phases with $A=\mathrm{Co}, \mathrm{Rh}$, and Ir and to investigate the role played by the transition metal species in determining the electronic structure and transport properties.

\section{METHODOLOGY}

\section{Electronic structure calculations}

Density functional theory (DFT) calculations were performed using the Quantum Espresso software. ${ }^{27}$ For the exchange and correlation, the Perdew-Burke-Enzerhof generalized gradient approximation functional was used. ${ }^{28}$ The core electrons were treated using ultrasoft pseudopotentials ${ }^{29}$ and the valence electrons were treated as plane waves with kinetic-energy cutoffs of $30 \mathrm{Ry}$ and $240 \mathrm{Ry}$ for the wave functions and charge density, respectively. The calculations were performed using an $8 \times 8 \times 8$ Monkhorst-Pack $k$-point mesh. The experimental lattice parameters and atomic positions from Refs. 20 and 21 were used as initial inputs. From these, the theoretical minimum energy lattice parameters were determined, and the internal atomic positions were relaxed using the Broyden-Fletcher-Goldfarb-Shanno method until the total forces on each atom were below $0.0004 \mathrm{Ry} / \mathrm{au}$ $(0.01 \mathrm{eV} / \AA)$. The effect of spin-orbit (SO) interactions was not included in our calculations. SO interactions have been shown to have a relatively minor influence on the band structure of $\mathrm{Co}$ and $\mathrm{Rh}$ based skutterudites. ${ }^{26,30,31}$ In contrast, SO effects have been shown to be more important in Ir-based skutterudites, but were not found to significantly influence the valence band. ${ }^{31}$ Since $p$-type transport is the focus of this paper, SO interactions were not considered critical. The Seebeck coefficients were estimated using solutions to the Boltzmann transport equations within the constant relaxation time approximation.

\section{Synthesis}

Equal stoichiometric amounts of Te (5N Corp., 99.999\%) and Sn (Alfa-Aersar, 99.999\%) shot were prereacted in an evacuated quartz ampoule in a furnace up to $1400 \mathrm{~K}$ and then briefly pulverized in a Spex mill. Ir powder (Alfa-Aesar, 99.99\%), Rh sponge (Alfa-Aesar, 99.95\%), and Co powder (Alpha-Aesar, 99.999\%) were milled in stoichiometric amounts with the crushed Te-Sn ingot between 15 min and $2 \mathrm{~h}$ in a WC Spex vials using a ball-to-mass ratio range between $2: 1$ and 5:1. The resulting powders were annealed between 36 and $72 \mathrm{~h}$ and at temperatures between $900 \mathrm{~K}$ and $1200 \mathrm{~K}$. All handling of powder was performed in an argon filled glove-box. Annealed powders were then consolidated in a uniaxial hot press under flowing Ar in graphite dies. Compacted samples densities were measured to be in excess of $95 \%$ of theoretical values.

\section{Characterization}

Powder X-ray diffraction (PXRD) data were collected using a Philips X'PERT MPD diffractometer $(\mathrm{Cu}-\mathrm{K} \alpha$ radiation) in reflection mode. The lattice parameter determination using $\mathrm{Si}$ as internal standard and Rietveld refinements were performed using WinCSD program package. ${ }^{32}$ Electrical and thermal transport properties were measured under vacuum up to $1000 \mathrm{~K}$. The electrical resistivity and Hall coefficient measurements were carried out using Van der Pauw technique under a reversible $1 \mathrm{~T}$ magnetic field using pressureassisted tungsten electrodes. ${ }^{33}$ The Seebeck coefficients of the samples were obtained using $\mathrm{W}-\mathrm{Nb}$ thermocouples by applying a temperature gradient across the sample to oscillate between $+/-7.5 \mathrm{~K} .{ }^{34}$ The thermal diffusivity, $D$, was measured with a Netzsch LFA 457 laser flash apparatus, and thermal conductivity was then calculated from $\kappa=D d C_{p}$, where $d$ is the Archimedes density and $C_{p}$ is the heat capacity at constant pressure. For $C_{p}$, the Dulong-Petit limit was employed, which might lead to underestimated $\kappa$ at high temperatures. Ultrasonic measurements were performed at room temperature to obtain the longitudinal and transverse sound velocities, with an uncertainty of $\sim 5 \%$.

\section{RESULTS AND DISCUSSION}

\section{Electronic structure calculations}

The theoretical lattice parameters obtained after relaxing the structure are $\sim 1 \%-2 \%$ larger than the experimental 
values reported in the literature. The electronic band structure and density of states are shown for each of the $A \mathrm{Sn}_{1.5} \mathrm{Te}_{1.5}$ compounds in Figure 2. All three compounds were found to have substantial band gaps; $0.43 \mathrm{eV}, 0.19 \mathrm{eV}$, and $0.41 \mathrm{eV}$ for $A=\mathrm{Co}, \mathrm{Rh}$, and $\mathrm{Ir}$, respectively. In their investigation of the $\operatorname{Co} X_{1.5} Y_{1.5}$ series, Volja et al. reported similarly large band gaps (ranging from 0.41 to $0.61 \mathrm{eV}$ ). These energy gaps are significantly larger than those calculated for binary $\mathrm{ASb}_{3}$ skutterudites, which vary between $\sim 0.17 \mathrm{eV}$ for $A=$ Co to $0 \mathrm{eV}$ for $A=\mathrm{Rh}$ and $\mathrm{Ir}^{31}{ }^{31}$ Volja et al. attributed this difference to the flattening of the $t_{2 g}$-like states that form the valence band maximum, possibly due to increased bond polarity in the pnictogen-substituted compounds.

The valence band maximum in $A \mathrm{Sn}_{1.5} \mathrm{Te}_{1.5}$ compounds is characterized by a single light band at $\Gamma$, whereas the conduction band edge (also at $\Gamma$ ) contains two degenerate bands and a third band at slightly higher energy (between 0.04 and

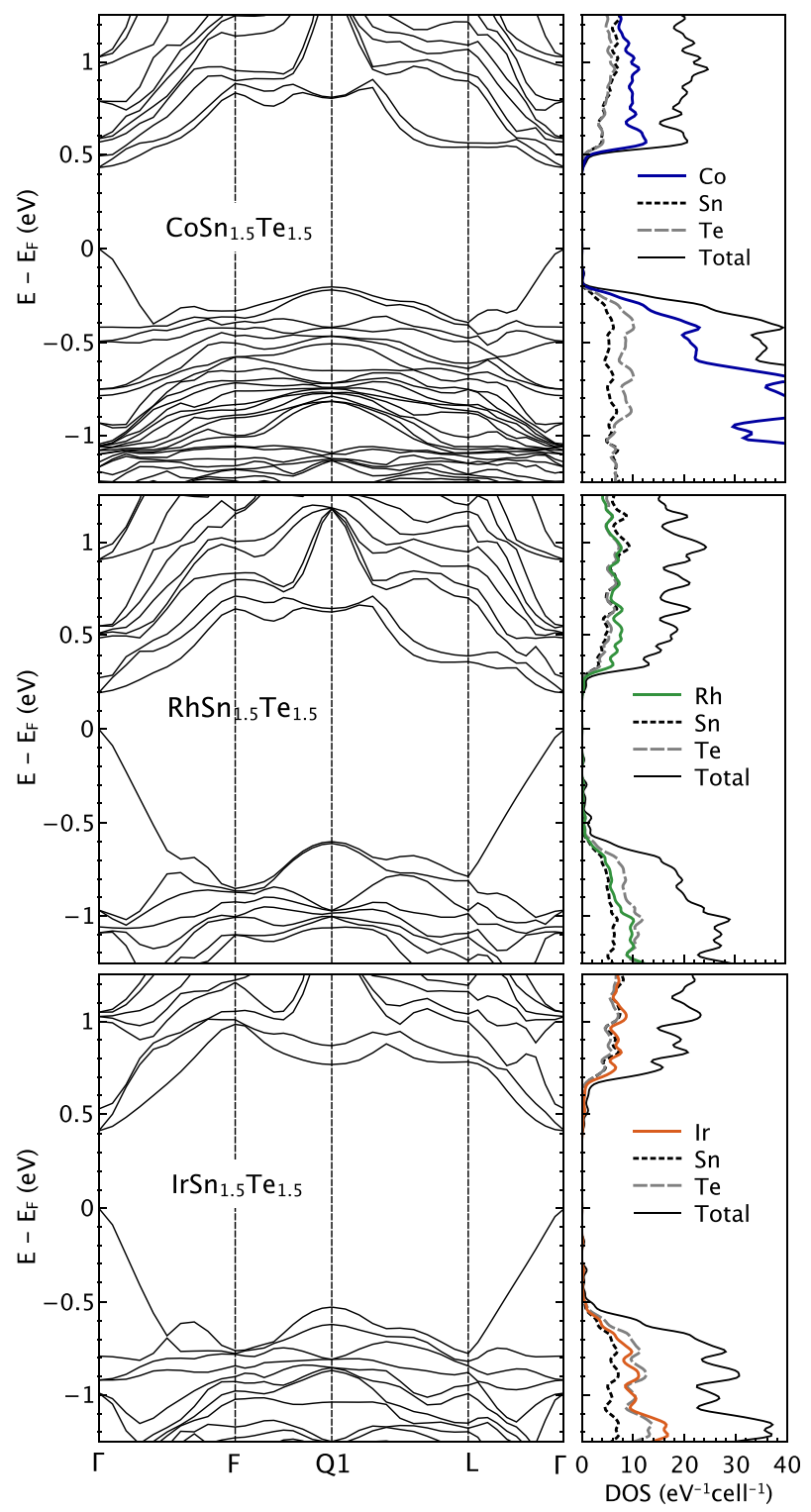

FIG. 2. Band structure (left) and total and partial density of states (right) for $A \mathrm{Sn}_{1.5} \mathrm{Te}_{1.5}$ compounds ( $A=\mathrm{Co}, \mathrm{Rh}, \mathrm{Ir}$ ). $\mathrm{E}-\mathrm{E}_{F}=0 \mathrm{eV}$ is set equal to the valence band maximum. The special $k$-space points were taken from Ref. 35 for space group $R \overline{3}$.
$0.12 \mathrm{eV}$ ). Similar to the binary skutterudites, the valence band in $A \mathrm{Sn}_{1.5} \mathrm{Te}_{1.5}$ has a Kane-like appearance for $A=\mathrm{Rh}$ and Ir. In contrast, the valence band in $\mathrm{CoSn}_{1.5} \mathrm{Te}_{1.5}$ appears to be far more parabolic than that of $\mathrm{CoSb}_{3}$, consistent with observations by Volja et al. ${ }^{26}$ The separation between the highest valence manifold (at $\Gamma$ ) and the next nearest band maxima (at $Q 1$ ) is significantly smaller in the pnictogen substituted skutterudites than in the binary analogues, opening possibilities for multi-band conduction in $p$-doped samples. In particular, in $\mathrm{CoSn}_{1.5} \mathrm{Te}_{1.5}$, the heavy bands with maxima at $Q 1$ are only $0.2 \mathrm{eV}$ below the valence band maximum. The contribution of the additional heavy bands to the density of states is apparent in the right hand panels of Figure 2. Here, it is clear that $\operatorname{CoSn}_{1.5} \mathrm{Te}_{1.5}$ has the highest density of states near the valence band edge.

The density of states is shown over a wider energy range in Figure 3 to help illustrate the influence of the $A$ site. The lowest energy region of the DOS ( -9 to $-6 \mathrm{eV}$ ) is mainly composed of bonding $\mathrm{Sn}$ and Te $s$ orbitals. The block between -6 and $0 \mathrm{eV}$ arises primarily from $t_{2 g}$ bonding states associated with the $A(\mathrm{Sn}, \mathrm{Te})_{6}$ octahedra, in which the $\mathrm{Sn}$ and Te $p$ orbitals hybridize with transition metal $d$ orbitals. Finally, the anti-bonding states associated with these hybridized bonds form the conduction band minimum $(0-3 \mathrm{eV}){ }^{31}$ Thus, changes to the $A$-site can be expected to influence both the valence and conduction band equally in skutterudite
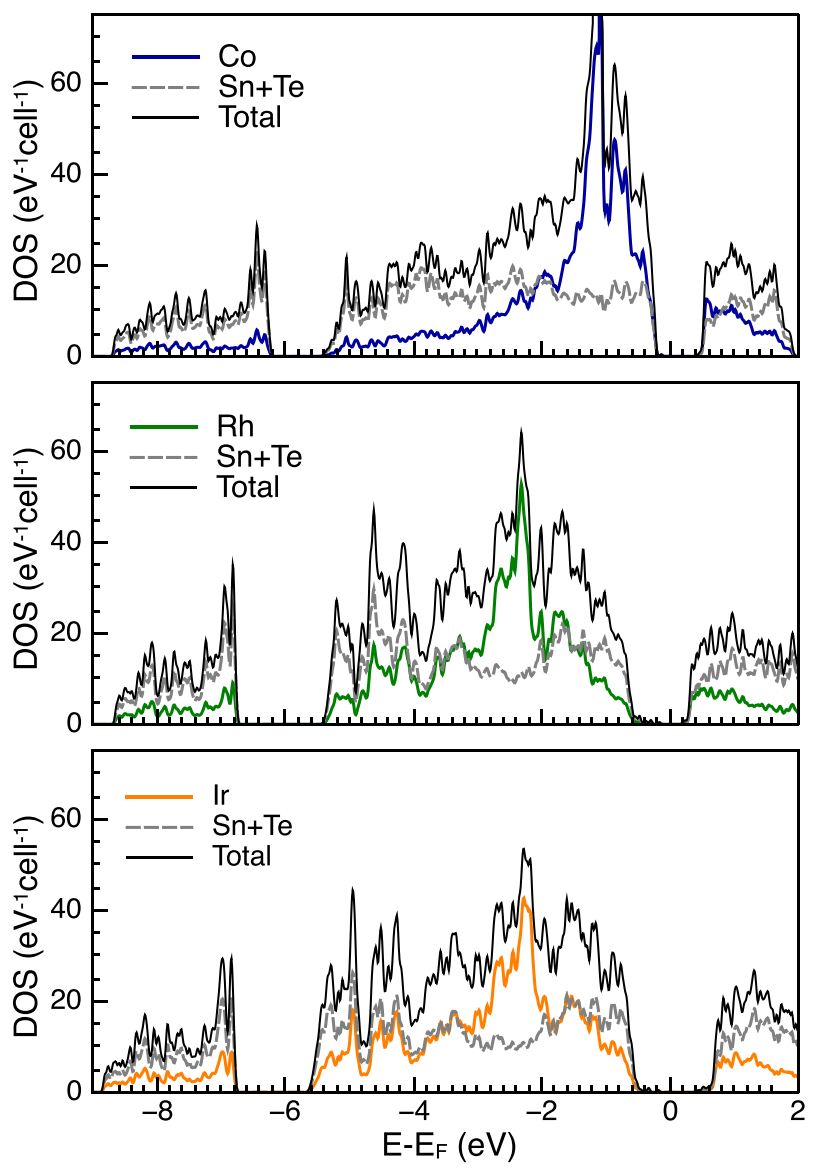

FIG. 3. Partial density of states comparing the $A$ contribution in $A \mathrm{Sn}_{1.5} \mathrm{Te}_{1.5}$ compounds $(A=\mathrm{Co}, \mathrm{Rh}, \mathrm{Ir}) . \mathrm{E}-\mathrm{E}_{F}=0 \mathrm{eV}$ is set equal to the valence band maximum. 
phases. In general, the Co $3 \mathrm{~d}$ orbitals are expected to be more localized spacially than the $\mathrm{Rh} 4 \mathrm{~d}$ or Ir $5 \mathrm{~d}$ contributions, which may explain the relatively non-disperse Co contribution between -2 and $0 \mathrm{eV}$ in Figure $3(\mathrm{a}) .^{30}$ The $A$ states become progressively more disperse for $A=\mathrm{Rh}$ and $A=\mathrm{Ir}$. Further, the electronegativity of $A(1.88,2.28$, and 2.20 on the Pauling scale for $\mathrm{Co}, \mathrm{Rh}$, and Ir, respectively) influences the relative position of the $A$ electronic states compared with the $\mathrm{Sn}$ and Te states. Due to its smaller electronegativity, the peak density of Co electronic states occurs approximately $1.2 \mathrm{eV}$ higher in energy relative to the peak densities of Ir or $\mathrm{Rh}$ states. These combined effects, which are also observed in binary $\mathrm{ASb}_{3}$ skutterudites, ${ }^{31}$ explain the very large density of states near the band gap in $\mathrm{CoSn}_{1.5} \mathrm{Te}_{1.5}$ compared with the $\mathrm{Rh}$ - and Ir-based compounds.

\section{Experimental}

\section{Phase analysis}

Bulk, hot pressed $A \mathrm{Sn}_{1.5} \mathrm{Te}_{1.5}$ samples with $A=\mathrm{Co}$, Rh, Ir were found to have high relative densities $(94.7 \%, 99.8 \%$, and $99.1 \%$ of theoretical, respectively) and were not reactive in air. PXRD patterns are shown in Figure 4. As expected, supercell reflections, resulting from the ordering of $\mathrm{Sn}$ and $\mathrm{Te}$ in the square rings were observed in all three compounds, consistent with a previous crystallographic study. ${ }^{21}$ From the PXRD patterns, it is apparent that the $\mathrm{RhSn}_{1.5} \mathrm{Te}_{1.5}$ and $\mathrm{IrSn}_{1.5} \mathrm{Te}_{1.5}$ are almost phase pure. In the former, a very small amount ( $<1$ wt. \%) of a secondary phase, possibly $\mathrm{RhTe}_{2}$, was observed, and in the latter, no impurity peaks were found. In contrast, the $\operatorname{CoSn}_{1.5} \mathrm{Te}_{1.5}$ sample contains a significant amount of SnTe (9wt. \% based on Rietveld refinements), as well as a very small amount of CoTe $(<1 \mathrm{wt} . \%)$. Significant quantities of binary telluride phases were also reported in a previous study of $A \mathrm{Sn}_{1.5} \mathrm{Te}_{1.5}$ samples that were made using a traditional solid state synthesis procedure. ${ }^{21}$ Rietveld refinements of $\mathrm{RhSn}_{1.5} \mathrm{Te}_{1.5}$ and $\mathrm{IrSn}_{1.5} \mathrm{Te}_{1.5}$ yields Ir and Rh occupancies of less than one $(\sim 0.96)$, while the Co site in $\mathrm{CoSn}_{1.5} \mathrm{Te}_{1.5}$ was found to be fully filled.

\section{Electronic transport}

The electronic transport properties, shown in Figure 5, were measured up to $873 \mathrm{~K}$ for $\mathrm{CoSn}_{1.5} \mathrm{Te}_{1.5}$ and $\mathrm{RhSn}_{1.5} \mathrm{Te}_{1.5}$ samples and up to $973 \mathrm{~K}$ for the $\mathrm{IrSn}_{1.5} \mathrm{Te}_{1.5}$ sample. This differentiation was motivated by the higher decomposition temperature of $\mathrm{IrSb}_{3}$ compared with $\mathrm{CoSb}_{3}$ reported by Fleurial et al. ${ }^{36}$ As shown in Figure 5(a), the behavior of the Hall carrier concentration differs strongly between the samples. In the $\mathrm{CoSn}_{1.5} \mathrm{Te}_{1.5}$ sample, $n_{H}$ is low and $p$-type at room temperature and increases exponentially with increasing temperature, indicative of non-degenerate semiconducting behavior. In contrast, the $\mathrm{RhSn}_{1.5} \mathrm{Te}$ and $\mathrm{IrSn}_{1.5} \mathrm{Te}_{1.5}$ samples exhibit high, relatively temperature independent $n_{H}$, characteristic of degenerate semiconductors. For all three compounds, assuming that the samples' stoichiometries are very close to the nominal $A \mathrm{Sn}_{1.5} \mathrm{Te}_{1.5}$ formula, non-degenerate semiconducting behavior is expected. However, small deviations from the nominal stoichiometry might be responsible for the observed

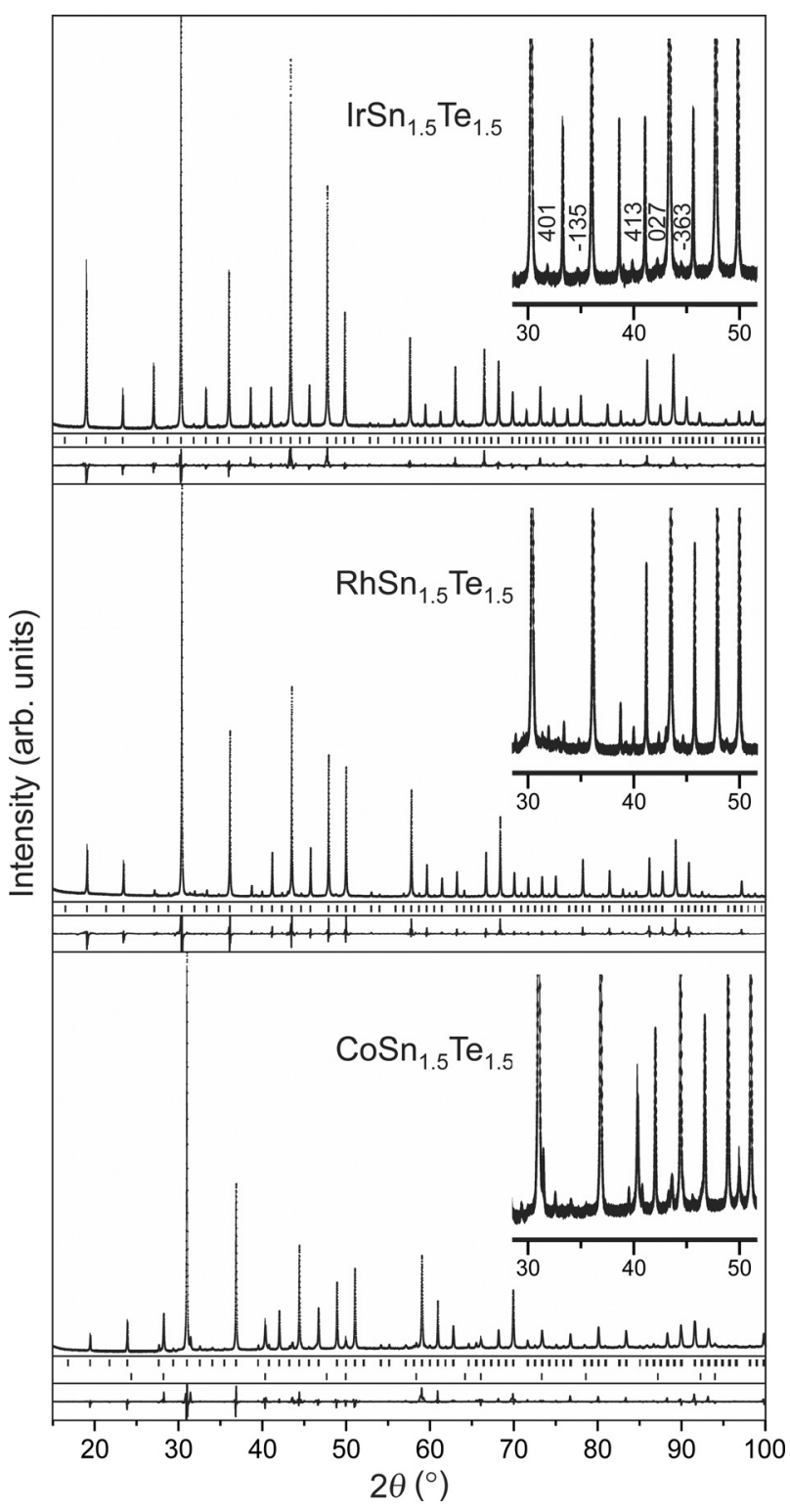

FIG. 4. PXRD patterns and Rietveld refinements of $A \mathrm{Sn}_{1.5} \mathrm{Te}_{1.5}$ samples ( $\mathrm{Cu}-\mathrm{K} \alpha 1$ radiation). The ticks mark the calculated reflection positions of the respective $A \mathrm{Sn}_{1.5} \mathrm{Te}_{1.5}$ phases from Ref. 21. For $\mathrm{CoSn}_{1.5} \mathrm{Te}_{1.5}$, a second phase, SnTe (lower ticks), was also included in the refinements. The difference profiles are shown underneath. The insets highlight the weak supercell reflections resulting from reduced symmetry.

degenerate $p$-type carrier concentrations in the Ir- and $\mathrm{Rh}-$ based samples. The refined occupancies for Ir and Rh occupancies of less than one $(\sim 0.96)$ might explain the observed $p$-type behavior. ${ }^{37}$ In contrast, Rietveld refinement of the Co site in $\mathrm{CoSn}_{1.5} \mathrm{Te}_{1.5}$ showed that site was fully filled, consistent with its low carrier concentration.

The resistivity, $\rho$, of the samples is shown in Figure 5(b). For $A=\mathrm{Co}$, the resistivity is high, and decreases with increasing temperature. A band gap, $E_{g}$, of $0.3 \mathrm{eV}$ was estimated from the resistivity in the high temperature intrinsic regime using $\rho \propto \exp \left(\frac{E_{g}}{2 k_{B} T}\right)$, as illustrated in the inset. This estimate is smaller than the energy gap predicted by DFT $(0.43 \mathrm{eV})$. For $A=\mathrm{Rh}$ and Ir, the resistivity shows a metallike temperature dependence, increasing with increasing temperature. 

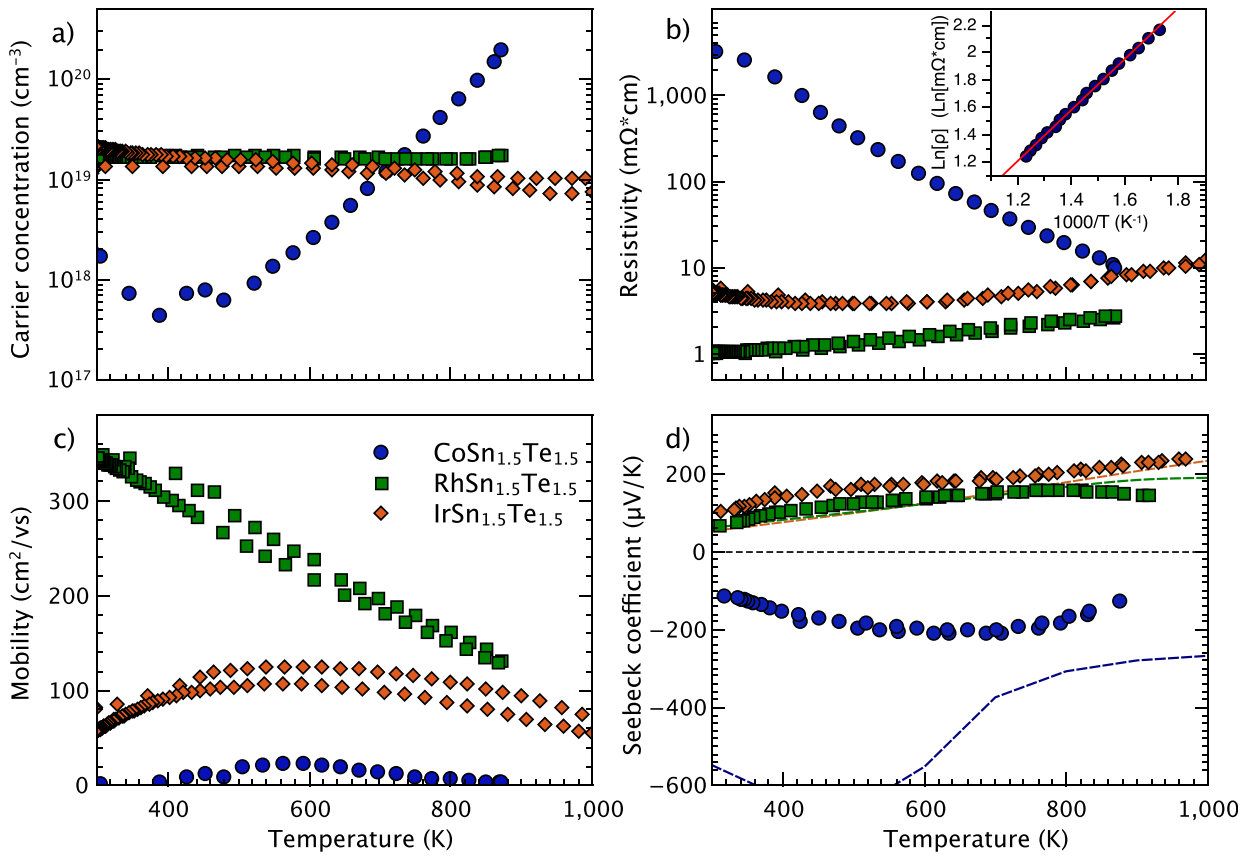

FIG. 5. (a) The Hall carrier concentration, (b) resistivity, (c) Hall mobility, and (d) Seebeck coefficients of $A \mathrm{Sn}_{1.5} \mathrm{Te}_{1.5}$ samples show degenerate and nondegenerate semiconducting behavior for $A=\mathrm{Ir}$, Rh and $A=\mathrm{Co}$, respectively. The experimental and predicted Seebeck coefficients show qualitative agreement.
The Hall mobility $\left(\mu_{H}=1 / n e \rho\right.$ ) of the samples is shown in Figure 5(c). $\mu_{H}$ is highest in the $\mathrm{RhSn}_{1.5} \mathrm{Te}$ sample, and decreases approximately with the $T^{-\nu}$ dependence expected when acoustic phonons are the primary scattering source (using $\nu=1.2$ ). The mobility of $\operatorname{IrSn}_{1.5} \mathrm{Te}_{1.5}$ is in very good agreement with previously reported results. ${ }^{6}$ In the Ir- and Co-based samples, $\mu_{H}$ exhibits two distinct temperature regimes. Above $600 \mathrm{~K}$, the mobility decreases with increasing temperature as seen in the $\mathrm{RhSn}_{1.5} \mathrm{Te}_{1.5}$ sample, but between 300 and $600 \mathrm{~K}$, the mobility appears to be suppressed by an additional scattering mechanism. In the $\mathrm{CoSn}_{1.5} \mathrm{Te}_{1.5}$ sample, $\mu_{H}$ is surprisingly low, which may be partly due to a failure of the single carrier type assumption in the relation $n_{H}=-1$ / $R_{H} \mathrm{e}$, where $R_{H}$ is the Hall coefficient. When mixed conduction is significant, as is the case for the $\mathrm{CoSn}_{1.5} \mathrm{Te}_{1.5}$ sample, $R_{H}$ goes to zero, causing $n_{H}$ to diverge towards infinity. The resulting overestimation of $n_{H}$ means that $\mu_{H}$ can be severely underestimated. ${ }^{38}$ Additionally, the $\mathrm{CoSn}_{1.5} \mathrm{Te}_{1.5}$ sample contains a large concentration of SnTe impurities, which may also contribute to its low $\mu_{H}$.

The Seebeck coefficients, $\alpha$, are shown in Figure 5(d). For $A=\mathrm{Rh}$ and $\mathrm{Ir}, \alpha$ increases with temperature, consistent with the degenerate $p$-type carrier concentrations. Room temperature effective masses of $0.25 m_{e}$ and $0.40 m_{e}$, respectively, were estimated using a single parabolic band (SPB) model assuming that acoustic phonon scattering limits transport. The Seebeck coefficients of the $\mathrm{RhSn}_{1.5} \mathrm{Te}_{1.5}$ sample reach a broad maximum and begin to decrease above $800 \mathrm{~K}$, which yields a band gap of $0.25 \mathrm{eV}$ using $E_{g}=2 \alpha_{\max } T_{\max }$. This estimate also is smaller than the DFT band gap. It should be noted, however, that band gap estimates obtained from either $\alpha$ or $\rho$ assume that holes and electrons have the same mobility, which is not likely the case here. In the $\mathrm{IrSn}_{1.5} \mathrm{Te}_{1.5}$ sample, $\alpha$ continues to increase linearly within the measured temperature range, consistent with results published in Ref. 6, indicating that it has a larger band gap than $\mathrm{RhSn}_{1.5} \mathrm{Te}_{1.5}$. These results are qualitatively consistent with the Seebeck coefficients predicted from the calculated electronic structures (assuming $n_{H}=2 \times 10^{19} h^{+} / \mathrm{cm}^{3}$ ), which are shown as dashed curves in Figure 5(d).

Figure 6 compares the experimental Seebeck coefficients of $\mathrm{RhSn}_{1.5} \mathrm{Te}_{1.5}$ and $\mathrm{IrSn}_{1.5} \mathrm{Te}_{1.5}$ at $800 \mathrm{~K}$ with the predicted values from DFT calculations and a single parabolic band model. The difference between the two models is striking, and confirms the strongly multi-band behavior expected based on the electronic band structures. The SPB model predicts optimized $z T$ at $n=10^{19} h^{+} / \mathrm{cm}^{3}$ for both $\mathrm{RhSn}_{1.5} \mathrm{Te}_{1.5}$ and $\operatorname{IrSn}_{1.5} \mathrm{Te}_{1.5}$. In other words, the current samples are already optimized, according to an SPB model. However, from Figure 6, it is clear that an SPB model underestimates the performance of samples with higher carrier concentrations, due to the large Seebeck contribution from the additional bands at Q1 in the valence band. We can thus infer

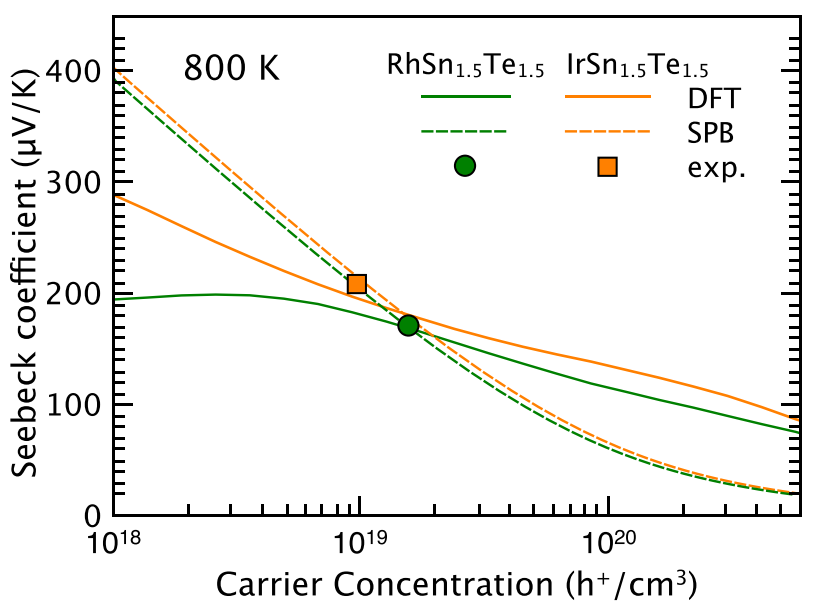

FIG. 6. A comparison of the calculated Seebeck coefficients (DFT) to a single parabolic band model (SPB) at $800 \mathrm{~K}$ illustrates the influence of multiband behavior in the valence band in $\mathrm{RhSn}_{1.5} \mathrm{Te}_{1.5}$ and $\mathrm{IrSn}_{1.5} \mathrm{Te}_{1.5}$ (SPB band masses are $0.24 m_{e}$ and $0.26 m_{e}$, respectively). The Co analogue was not included, due to mixed-conduction behavior dominating the transport. 
that higher carrier concentrations are still necessary to optimize the figure of merit.

The $\mathrm{CoSn}_{1.5} \mathrm{Te}_{1.5}$ sample has negative Seebeck coefficients, apparently contradicting the positive Hall coefficients. Such contradictory properties can often be observed in mixed-conduction systems, in particular, when the difference between the hole and electron effective mass is large. In $\mathrm{CoSn}_{1.5} \mathrm{Te}_{1.5}$, the light band at the valence band edge contributes disproportionately to the Hall coefficient, while the three heavier conduction bands dominate the Seebeck coefficient. The negative Seebeck coefficients suggest that the Fermi level is actually in the conduction band, despite the experimental $p$-type carrier concentrations. ${ }^{39} \mathrm{~A}$ small $n$-type carrier concentration of $n_{H}=5 \times 10^{17} \mathrm{~h}^{+} / \mathrm{cm}^{3}$ was chosen to calculate the Seebeck coefficients from the electronic structure (dashed curve). The predicted values severely overestimate the magnitude in this case, which may stems in part from the difficulty of accurately measuring $n_{H}$ in mixed carrier type systems. A previous report on $\mathrm{CoSn}_{1.5} \mathrm{Te}_{1.5}$ by Nagamoto et al. also evidenced mixed conduction. Nagamoto reported high resistivity, negative Seebeck coefficients, and near-zero Hall mobility. ${ }^{40}$ However, they observed $n$-type Hall coefficients, suggesting that their samples were weighted towards an electron deficient stoichiometry. This small difference in the chemical potential resulted in much higher $n$-type Seebeck coefficients at room temperature, comparable to the calculated Seebeck coefficients in the current study. We note also that the presence of SnTe as an impurity phase may also have an influence on the experimental properties, causing them to deviate from the calculated ones.

\section{Thermal transport}

The total thermal conductivity, $\kappa$, of $A \mathrm{Sn}_{1.5} \mathrm{Te}_{1.5}$ samples is shown in Figure 7(a). The electronic contribution to the thermal conductivity, $\kappa_{e}$, was estimated using the Wiedemann-Franz relation, $\kappa_{e}=L T / \rho$, where the temperature dependent Lorenz numbers, $L$, were determined from an SPB model using the experimental Seebeck coefficients (see inset of Fig. 7(a)). ${ }^{41}$ Subtracting $\kappa_{e}$ from $\kappa$ yields the combined lattice and bipolar contributions, $\kappa_{L}+\kappa_{B}$, shown in Figure 7(b). The bipolar contribution is significant only in the $\mathrm{CoSn}_{1.5} \mathrm{Te}_{1.5}$ sample above $600 \mathrm{~K}$.

Comparing the lattice thermal conductivity of $A \mathrm{Sn}_{1.5} \mathrm{Te}_{1.5}$ samples at room temperature, it becomes apparent that $\kappa_{L}$ does not follow the trend expected based solely on differences in the theoretical densities, $d$, of the samples. Even though $\mathrm{CoSn}_{1.5} \mathrm{Te}_{1.5}$ is the lightest of the three compounds, its $\kappa_{L}$ is the lowest. This can be explained in part by the transverse and longitudinal sound velocities $\left(\nu_{T}\right.$ and $\nu_{L}$, respectively), and the shear and bulk elastic moduli ( $G$ and $K$, respectively), given in Table I. Although the $\mathrm{CoSn}_{1.5} \mathrm{Te}_{1.5}$ sample has the lowest theoretical density, it does not have the highest speed of sound. The $\mathrm{RhSn}_{1.5} \mathrm{Te}_{1.5}$ sample stands out as being the stiffest of the three compounds, leading to higher sound velocity in $\mathrm{RhSn}_{1.5} \mathrm{Te}_{1.5}$ than in $\mathrm{CoSn}_{1.5} \mathrm{Te}_{1.5}$. It is also likely that additional phonon scattering, possibly caused by SnTe impurities, reduces $\kappa_{L}$ at room temperature in the Cobased sample.
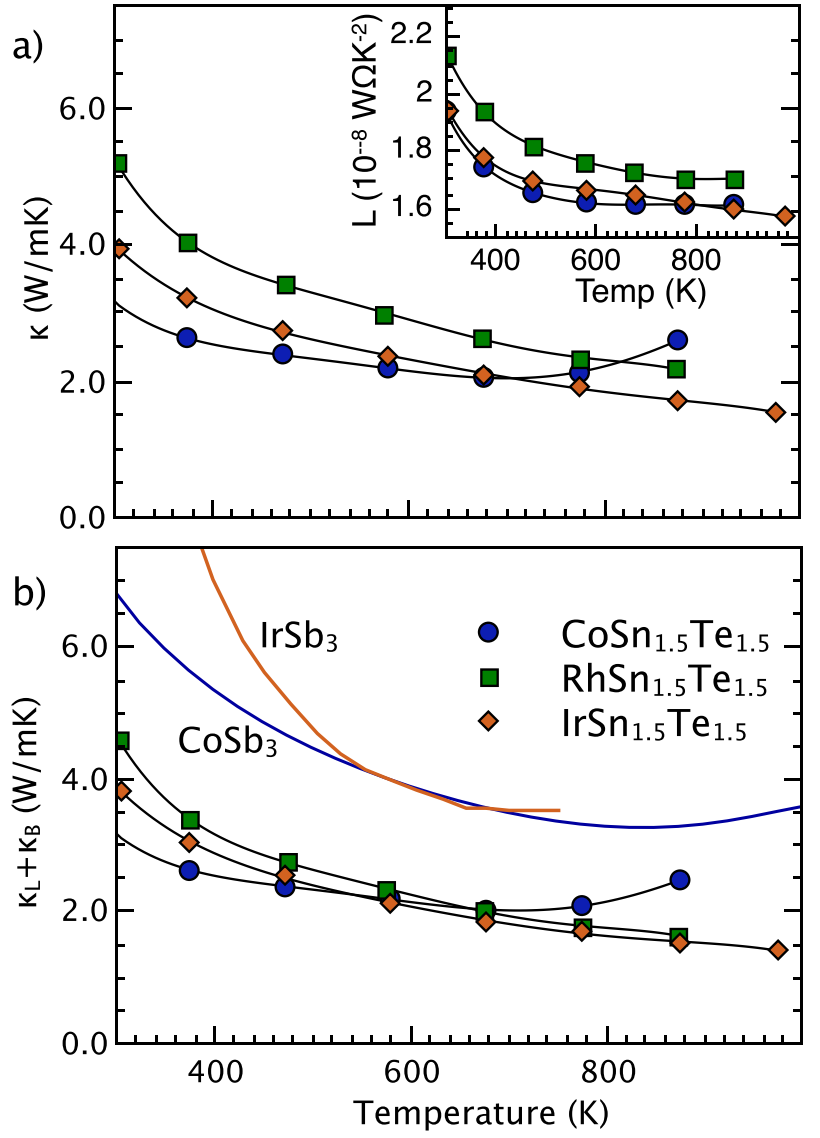

FIG. 7. (a) Total and (b) lattice and bipolar thermal conductivity of $A \mathrm{Sn}_{1.5} \mathrm{Te}_{1.5}$ samples. $\mathrm{CoSb}_{3}$ and $\mathrm{IrSb}_{3}$ data taken from Ref. 6. Inset: The Lorenz numbers were calculated within a single parabolic band model.

Compared with binary skutterudite phases $\mathrm{CoSb}_{3}$ and $\mathrm{IrSb}_{3}$, shown as the solid curves in Figure 7(b), ${ }^{6} \kappa_{L}$ in pnictogen-substituted skutterudites is reduced by approximately $50 \%$. This can be attributed to the larger unit cell size (32 atoms in ternary compounds compared with 16 in binaries) and also from possible disorder on the $\mathrm{Sn}$ and $\mathrm{Te}$ sites. Despite the reduction of $\kappa_{L}$ compared with $\mathrm{ASb}_{3}$ compounds, at high temperatures, Umklapp scattering alone proves insufficient to reduce $\kappa_{L}$ to the predicted minimum lattice thermal conductivity, $\kappa_{\min }$, given for each compounds in Table I. Here, $\kappa_{\min }$ was calculated using Eq. (1), ${ }^{12,42}$ using the longitudinal and transverse sound velocities given in Table I. This suggests that additional strategies should be employed to further reduce $\kappa_{L}$ and improve the figure of merit.

$$
\kappa_{\min }=\frac{1}{2}\left(\frac{\pi}{6}\right)^{1 / 3} k_{B} V^{-2 / 3}\left(2 \nu_{T}+\nu_{L}\right)
$$

TABLE I. $d$ is the theoretical density, $G$ and $K$ are the shear and bulk elastic moduli, $\nu_{L}$ and $\nu_{T}$ are the longitudinal and transverse sound velocities, and $\kappa_{\min }$ is the minimum $\kappa_{L}$ at $\mathrm{T}>\Theta_{D}$.

\begin{tabular}{lcccccc}
\hline & $d\left(\mathrm{~g} / \mathrm{cm}^{3}\right)$ & $G(\mathrm{GPa})$ & $K(\mathrm{GPa})$ & $\nu_{T}(\mathrm{~m} / \mathrm{s})$ & $\nu_{L}(\mathrm{~m} / \mathrm{s})$ & $\kappa_{\min }(\mathrm{W} / \mathrm{mK})$ \\
\hline $\mathrm{Co}$ & 7.46 & 47 & 70 & 2510 & 3080 & 0.33 \\
$\mathrm{Rh}$ & 7.80 & 51 & 94 & 2570 & 3230 & 0.30 \\
$\mathrm{Ir}$ & 8.73 & 48 & 79 & 2340 & 2910 & 0.27 \\
\hline \hline
\end{tabular}




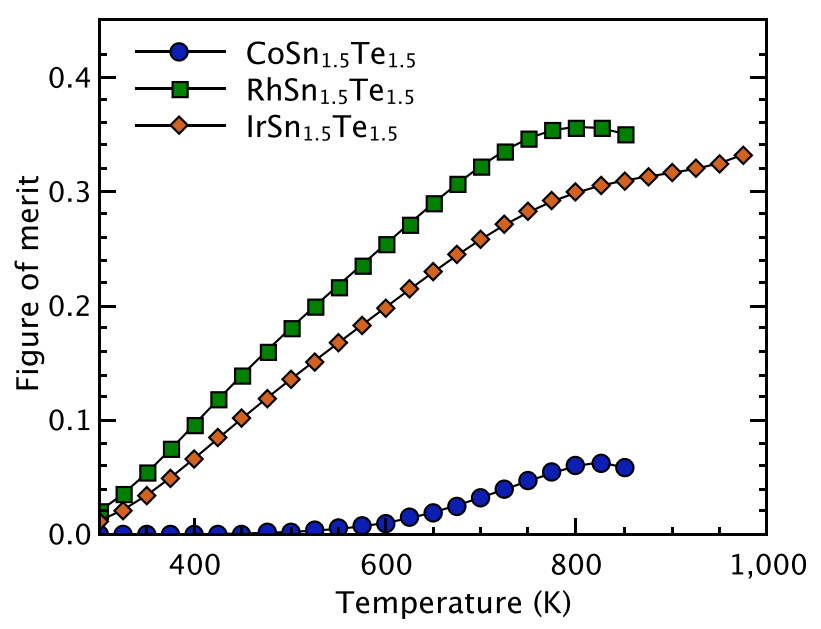

FIG. 8. The figure of merit is highest for $A=\mathrm{Ir}, \mathrm{Rh}$, due to the relatively high carrier concentrations. In contrast, the low $z T$ values in $\operatorname{CoSn}_{1.5} \mathrm{Te}_{1.5}$ are due to mixed $n$ - and $p$-type conduction.

\section{Figure of merit}

The thermoelectric figure of merit of $A \mathrm{Sn}_{1.5} \mathrm{Te}_{1.5}$ samples is shown in Figure 8. The $\operatorname{CoSn}_{1.5} \mathrm{Te}_{1.5}$ sample has very low $z T$ values due to its large resistivity and mixed $n$ - and $p$ type conduction. The $\mathrm{RhSn}_{1.5} \mathrm{Te}_{1.5}$ and $\mathrm{IrSn}_{1.5} \mathrm{Te}_{1.5}$ samples have peak $z T$ values of $\sim 0.35$ at $800 \mathrm{~K}$ and $1000 \mathrm{~K}$, respectively. Although these values are modest, further optimization of the electronic and thermal properties via doping or filling may lead to significant improvements. In both ternary and binary skutterudites, the primary factor limiting the $z T$ in $p$-type skutterudites is the valence band structure, which is characterized by a single light band. In $n$-type skutterudites, higher $z T$ is the result of the three degenerate bands that form the conduction band edge. Here also, we expect that $n$ type doping could lead to significantly higher $z T$ values, particularly in the $\mathrm{CoSn}_{1.5} \mathrm{Te}_{1.5}$, which benefits from a very high density of states and a high band degeneracy.

\section{CONCLUSION}

Pnictogen substituted skutterudites with compositions of $A \mathrm{Sn}_{1.5} \mathrm{Te}_{1.5}(A=\mathrm{Co}, \mathrm{Rh}, \mathrm{Ir})$ were shown to be semiconducting compounds with significantly larger band gaps than their binary analogues. The $A$ element was shown to have a strong influence on the density of states near the band edge, with the largest density of states found in $\mathrm{CoSn}_{1.5} \mathrm{Te}_{1.5}$. High temperature electronic transport measurements confirmed these results, revealing non-degenerate semiconducting behavior for $A=\mathrm{Co}$, and degenerate $p$-type behavior for $A=\mathrm{Rh}$ and Ir. Maximum $z T$ values of 0.35 were observed in $A=\mathrm{Rh}$ and Ir samples. However, with low thermal conductivity, reasonably large band gaps, and band structure features closely resembling that of binary skutterudites, it appears that the potential of these pnictogen substituted skutterudites is limited only by the ability to control their carrier concentrations.

\section{ACKNOWLEDGMENTS}

This research was carried out in part at the Jet Propulsion Laboratory, California Institute of Technology, under a contract with the National Aeronautics and Space Administration and was supported by the NASA Science Missions Directorate's Radioisotope Power Systems Technology Advancement Program. We gratefully acknowledge Gregory Gerig for performing Seebeck measurements. Financial assistance of the Scientific and Technological Research Council of Turkey and NASA Postdoctoral Fellowship Program are acknowledged by A.U. and A. Z., respectively.

${ }^{1}$ L. E. Bell, "Cooling, heating, generating power, and recovering waste heat with thermoelectric systems," Science 321, 1457 (2008).

${ }^{2}$ G. J. Snyder and E. S. Toberer, "Complex thermoelectric materials," Nature Mater. 7, 105-114 (2008).

${ }^{3}$ J. R. Sootsman, D. Y. Chung, and M. G. Kanatzidis, "New and old concepts in thermoelectric materials," Angew. Chem., Int. Ed. Engl 48(46), 8616-8639 (2009).

${ }^{4}$ F. J. DiSalvo, "Thermoelectric cooling and power generation," Science 285(5428), 703-706 (1999).

${ }^{5}$ C. Uher, "Skutterudites: Prospective novel thermoelectrics," in Semiconductors and Semimetals (Academic Press, 2001), Vol. 69

${ }^{6}$ J.-P. Fleurial, T. Caillat, and A. Borshchevsky, "Skutterudites: An update," in Proceedings of XVI International Conference on Thermoelectrics (International Thermoelectric Society, 1997).

${ }^{7}$ G. S. Nolas, G. Fowler, and J. Yang, "Assessing the role of filler atoms on the thermal conductivity of filled skutterudites," J. Appl. Phys. 100(4), 043705 (2006).

${ }^{8}$ P. F. Qui, J. Yang, R. H. Liu, X. Shi, X. Y. Huang, G. J. Snyder, W. Zhang, and L. D. Chen, "High-temperature electrical and thermal transport properties of fully filled skutterudites $R \mathrm{Fe}_{4} \mathrm{Sb} 12(R=\mathrm{Ca}, \mathrm{Sr}, \mathrm{Ba}, \mathrm{La}, \mathrm{Ce}$, Pr, Nd, Eu, and Yb)," J. Appl. Phys. 109, 063713 (2011).

${ }^{9}$ B. Sales, D. Mandrus, B. C. Chakoumakos, V. Keppens, and J. R. Thompson, "Filled skutterudite antimonides: Electron crystals and phonon glasses," Phys. Rev. B 56, 15081 (1997).

${ }^{10}$ M. M. Koza, M. R. Johnson, R. Viennois, H. Mutka, L. Girard, and D. Ravot, "Breakdown of phonon glass paradigm in la- and ce-filled fe4sb12 skutterudites," Nature Mater. 7(10), 805-810 (2008).

${ }^{11}$ M. Christensen, A. B. Abrahamsen, N. B. Christensen, F. Juranyi, N. H. Andersen, K. Lefmann, J. Andreasson, C. R. Bahl, and B. B. Iversen, "Avoided crossing of rattler modes in thermoelectric materials," Nature Mater. 7(10), 811-815 (2008).

${ }^{12}$ E. S. Toberer, A. Zevalkink, and G. J. Snyder, "Phonon engineering through crystal chemistry," J. Mater. Chem. 21(40), 15843 (2011).

${ }^{13}$ V. Keppens, D. Mandrus, B. Sales, B. C. Chakoumakos, P. Dai, R. Coldea, M. B. Maple, D. A. Gajewski, A. J. Freeman, and S. Bennington, "Localized vibrational modes in metallic solids," Nature 395, 876-878 (1998).

${ }^{14}$ Y. Z. Pei, J. Yang, L. D. Chen, W. Zhang, J. R. Salvador, and J. Yang, "Improving thermoelectric performance of caged compounds through light-element filling," Appl. Phys. Lett. 95(4), 042101 (2009).

${ }^{15}$ G. Nolas, M. Kaeser, R. T. Littleton IV, and T. M. Tritt, "High figure of merit in partially filled ytterbium skutterudite materials," Appl. Phys. Lett. 77, $1855(2000)$

${ }^{16}$ P. Vaqueiro, G. G. Sobany, A. V. Powelll, and K. S. Knight, "Structure and thermoelectric properties of the ordered skutterudite $\mathrm{CoGe}_{1.5} \mathrm{Te}_{1.5}$," J. Solid State Chem. 179, 2047-2053 (2006).

${ }^{17}$ P. Vaqueiro and G. G. Sobany, "Ternary skutterudites: Anion ordering and thermoelectric properties," in MRS Fall Meeting (Mater. Res. Soc. Symp. Proc., 2007).

${ }^{18}$ J. Navratil, T. Plechacek, L. Benes, C. Drasar, and F. Laufek, "Thermoelectric properties of $\mathrm{Co}_{4} \mathrm{Sn}_{6} \mathrm{Se}_{6}$ ternary skutterudites," J. Electron. Mater. 39(9), 1880-1884 (2010).

${ }^{19}$ J. Navratil, T. Plechacek, L. Benes, and M. Vlcek, "Some transport properties of $\mathrm{CoGe}_{1.5} \mathrm{Te}_{1.5}$," Chalcogenide Lett. 1(6), 73-77 (2004).

${ }^{20}$ R. J. Cava and J. W. G. Bos, "Synthesis, crystal structure and thermoelectric properties of $\operatorname{IrSn}_{1.5} \mathrm{Te}_{1.5}$-based skutterudites," Solid State Commun. 141, 38-41 (2007).

${ }^{21}$ P. Vaqueiro, G. G. Sobany, and A. V. Powelll, "A synchrotron powder Xray diffraction study of the skutterudite-related phases $A B_{1.5} \mathrm{Te}_{1.5}(A=\mathrm{Co}$, Rh, Ir; $B=\mathrm{Ge}, \mathrm{Sn})$,” Dalton Trans. 39, 1020-1026 (2010). 
${ }^{22}$ J. Navratil, T. Plechacek, C. Drasar, M. Vlcek, L. Benes, and F. Laufek, "Effect of partial La filling of $\mathrm{Co}_{4} \mathrm{Ge}_{6} \mathrm{Te}_{6}$ ternary skutterudite on their thermoelectric properties," in 6th European Conference on Thermoelectrics, 2009.

${ }^{23}$ G. S. Nolas, J. Yang, and R. W. Ertenberg, "Transport properties of CoGe $e_{1.5} \mathrm{Se}_{1.5}$, " Phys. Rev. B 68, 193206 (2003).

${ }^{24}$ G. S. Nolas, M. Beekman, R. W. Ertenberg, and J. Yang, "Low temperature transport properties of Ni-doped $\mathrm{CoGe}_{1.5} \mathrm{Se}_{1.5}$," J. Appl. Phys. 100, 036101 (2006).

${ }^{25}$ Y. Dong, K. Wei, and G. S. Nolas, "Transport properties of partially filled skutterudite derivatives $\mathrm{Ce}_{0.13} \mathrm{Co}_{4} \mathrm{Ge}_{6} \mathrm{Se}_{6}$ and $\mathrm{Yb}_{0.14} \mathrm{Co}_{4} \mathrm{Ge}_{6} \mathrm{Se}_{6}$," Phys. Rev. B 87, 195203 (2013).

${ }^{26}$ D. Volja, B. Kozinsky, A. Li, D. Wee, N. Marzari, and M. Fornari, "Electronic, vibrational, and transport properties of pnictogen-substituted ternary skutterudites," Phys. Rev. B 85, 245211 (2012).

${ }^{27}$ P. Giannozzi, S. Baroni, N. Bonini, M. Calandra, R. Car, C. Cavazzoni, D. Ceresoli, G. Chiarotti, M. Cococcioni, I. Dabo, A. Corso, D. Gironcoli, D. Fabris, G. Fratesi, R. Gebauer, U. Gerstmann, C. Gougoussis, A. Kokalj, M. Lazzeri, L. Martin-Samos, N. Marzari, F. Mauri, R. Mazzarello, S. Paolini, A. Pasquarello, L. Paulatto, C. Sbraccia, S. Scandolo, G. Sclauzero, A. Seitsonen, A. Smogunov, P. Umari, and R. Wentzcovitch, J. Phys.: Condens. Matter 21, 395502 (2009).

${ }^{28}$ J. P. Perdew, K. Burke, and M. Ernzerhof, "Generalized gradient approximation made simple," Phys. Rev. Lett. 77, 3865-3868 (1996).

${ }^{29} \mathrm{D}$. Vanderbilt, "Soft self-consistent pseudopotentials in a generalized eigenvalue formalism," Phys. Rev. B 41, 7892-7895 (1990).

${ }^{30}$ D. J. Singh and W. E. Pickett, "Skutterudite antimonides: Quasilinear bands and unusual transport,” Phys. Rev. B 50, 11235-11238 (1994).

${ }^{31}$ K. Koga, K. Akai, K. Oshiro, and M. Matsuura, "Electronic structure and optical properties of binary skutterudite antimonides," Phys. Rev. B 71, 155119 (2005).
${ }^{32}$ L. Akselrud and Y. Grin, "Wincsd: Software package for crystallographic calculations (version 4)," J. Appl. Crystallogr. 47, 803-805 (2014).

${ }^{33}$ K. A. Borup, E. S. Toberer, L. Zoltan, G. Nakatsukasa, M. Errico, J. Fleurial, B. Iverson, and G. J. Snyder, "Measurement of the electrical resistivity and hall coefficient at high temperatures," Rev. Sci. Instrum. 83, 123902 (2012).

${ }^{34}$ C. Wood, D. Zoltan, and G. Stapfer, "Measurement of Seebeck coefficient using a light pulse,” Rev. Sci. Instrum. 56, 719 (1985).

${ }^{35} \mathrm{~W}$. Setyawan and S. Curtarolo, "High-throughput electronic band structure calculations: Challenges and tools," Comput. Mater. Sci. 49, 299-312 (2010).

${ }^{36}$ T. Calliat, A. Borshchevsky, and J. P. Fleurial, "Properties of single crystalline semiconducting cosb3," J. Appl. Phys. 80, 4442-4449 (1996).

${ }^{37}$ A. Zevalkink, W. G. Zeier, E. Cheng, G. J. Snyder, J.-P. Fleurial, and S. $\mathrm{K}$. Bux, "Nonstoichiometry in the Zintl phase $\mathrm{Yb}_{1-\delta} \mathrm{Zn}_{2} \mathrm{Sb}_{2}$ as a route to thermoelectric optimization," Chem. Mater. 26, 5710-5717 (2014).

${ }^{38}$ A. Zevalkink, G. S. Pomrehn, S. Johnson, J. Swallow, Z. M. Gibbs, and G. J. Snyder, "Influence of the triel elements $(M=\mathrm{Al}, \mathrm{Ga}, \mathrm{In})$ on the transport properties of $\mathrm{Ca}_{5} M_{2} \mathrm{Sb}_{6}$ Zintl compounds," Chem. Mater. 24(11), 2091-2098 (2012).

${ }^{39}$ J. Schmitt, Z. Gibbs, G. J. Snyder, and C. Felser, "Resolving the true band gap of $\mathrm{ZrNiSn}$ half-heusler thermoelectric materials," Mater. Horizons 2 , 68-75 (2015)

${ }^{40}$ Y. Nagamoto, K. Tanaka, and T. Koyanagi, "Thermoelectric properties of the skutterudite-related phase $\mathrm{CoSn}_{1.5} \mathrm{Te}_{1.5}$," in Proceedings of XVI International Conference on Thermoelectrics (1997), pp. 330-333.

${ }^{41}$ A. F. May, E. S. Toberer, A. Saramat, and G. J. Snyder, "Characterization and analysis of thermoelectric transport in n-type $\mathrm{Ba}_{8} \mathrm{Ga}_{16-x} \mathrm{Ge}_{30+\mathrm{x}}$," Phys. Rev. B 80, 125205 (2009).

${ }^{42}$ D. Cahill, S. Watson, and R. Pohl, "Lower limit to the thermal conductivity of disordered crystals," Phys. Rev. B 46(10), 6131-6140 (1992). 